\title{
Physical Function in Critically Ill Patients during the Duration of ICU and Hospital Admission
}

\author{
Damini R Aglawe ${ }^{1} \odot$, Bela M Agarwal ${ }^{2} \odot$ B Bhoomika D Sawant $^{3} \odot$
}

\begin{abstract}
Background: Impaired physical activity and functional ability is a significant problem in critical illness survivors. Measurement of physical functioning through intensive care unit (ICU) stay determines patients at risk of poor physical outcomes, monitors efficacy of intervention, and informs recovery trajectories.

Objectives: Study objective was to assess physical function trajectory and identify residual functional limitations in critically ill patients admitted to ICU at the point of discharge from the hospital using robust clinical measures.

Materials and methods: Following ethical approval, 100 patients (78 males and 22 females) admitted to medical and surgical ICUs were recruited. Scores on Functional Status Score in ICU (FSS-ICU), Physical Function ICU Test (PFIT), and Functional Independence Measure (FIM) were recorded. Day of physiotherapy reference in the ICU was considered as day of ICU admission. Data were collected at three points, namely ICU admission, ICU discharge, and hospital discharge.

Results: Scores on all outcome measures increased linearly, and an upward functional trajectory was observed in patients from the point of ICU admission till hospital discharge $(p>0.001)$.

Conclusion: Deficits in functional recovery exist until hospital discharge, substantiating the need to implement home-based rehabilitation to recover optimum physical function and independence in activities of daily living.

Keywords: Critical illness, Intensive care units, Physical function, Physiotherapy.

Indian Journal of Critical Care Medicine (2022): 10.5005/jp-journals-10071-24144
\end{abstract}

\section{INTRODUCTION}

Physical activity is key to healthy functioning of the body. ${ }^{1}$ Physical function refers to the ability to carry out various activities ranging from self-care to more vigorous activities requiring increased degrees of mobility, strength, or endurance. Regular moderate physical activity has favorable effect on all systems of the body. Lack of regular physical activity results in dysfunction of body systems, impaired physical functional ability, and poor quality of life. ${ }^{2}$

Patients in ICUs need continuous intensive or invasive monitoring; support of airways, breathing, or circulation; stabilization of acute or life-threatening medical problems; comprehensive management of injury and/or illness; and maximization of comfort. Patients in the ICU with critical illness are exposed to prolonged bed rest and immobilization, leading to decreased physical activity and loss of functional abilities and dysfunctions of organ systems of the body. Impact of inactivity in the form of bed rest on musculoskeletal and cardiovascular systems is central to loss of functional independence. ' Skeletal muscle atrophy, loss of muscle contractility and strength, and reduced bone mass due to increased rate of bone resorption than bone formation take place in the musculoskeletal system. ${ }^{3}$ Response of cardiac muscles to inactivity is similar to the response of skeletal muscles. Cardiac muscle atrophy along with reduced stroke volume, increased resting heart rate, and reduced cardiac capacity occurs in response to physical activity. ${ }^{4}$ Signs of orthostatic intolerance begin to develop after 3-4 days of bed rest. Increase in orthostatic intolerance and decrease in muscle strength together lead to a higher risk of fall and injury. ${ }^{5}$

Measuring physical functioning early and longitudinally in the ICU is key to determining patients at high risk of poor physical

\footnotetext{
${ }^{1-3}$ Department of Physiotherapy, MGM School of Physiotherapy, MGM Institute of Health Sciences, Navi Mumbai, Maharashtra, India

Corresponding Author: Bela M Agarwal, Department of Physiotherapy, MGM School of Physiotherapy, MGM Institute of Health Sciences, Navi Mumbai, Maharashtra, India, Phone: +91 9819000674, e-mail: belaagarwal@gmail.com

How to cite this article: Aglawe DR, Agarwal BM, Sawant BD. Physical Function in Critically III Patients during the Duration of ICU and Hospital Admission. Indian J Crit Care Med 2022;26(3):314-318.

Source of support: Nil

Conflict of interest: None
}

outcomes, monitoring intervention efficacy, and informing recovery trajectories. It is beneficial to knowing the current functional status and further planning of appropriate treatment for the individual. ${ }^{6}$ Assessment followed by initiation of early rehabilitation of patients during ICU stay helps to increase the success rate in ventilator weaning, reduce ICU and hospital stay, discharge early, and improve quality of life during and after ICU stay. ${ }^{7}$ Physical function is not completely recovered after ICU discharge; various physical, psychological, clinical, and environmental factors influence physical activity of hospitalized patients. ${ }^{8}$

ICU functional scales have been administered primarily for the evaluation of physical activity of patients during ICU stay. Application of these scales during the post-ICU hospital phase is inadequate, resulting in lack of evaluation of physical activity status of patients during the post-ICU hospital phase, despite the presence of residual impairments. There is lacuna in the literature indicating functional limitations faced by patients post-ICU discharge. Hence,

C) The Author(s). 2022 Open Access This article is distributed under the terms of the Creative Commons Attribution 4.0 International License (https://creativecommons. org/licenses/by-nc/4.0/), which permits unrestricted use, distribution, and non-commercial reproduction in any medium, provided you give appropriate credit to the original author(s) and the source, provide a link to the Creative Commons license, and indicate if changes were made. The Creative Commons Public Domain Dedication waiver (http://creativecommons.org/publicdomain/zero/1.0/) applies to the data made available in this article, unless otherwise stated. 
this study was designed to evaluate physical function recovery trajectory of patients during ICU stay until hospital discharge.

\section{Methodology}

Ethical approval was sought from the Institutional Ethical Committee. Patients of age-group 18-60 years admitted to medical and surgical ICUs from August to December 2020, with neurological, respiratory, medical and surgical conditions, and physiotherapy referral were included. Patients diagnosed with COVID-19 confirmed with reverse transcription polymerase chain reaction (RT-PCR) test and other infectious diseases, like acquired immunodeficiency syndrome (AIDS) and hepatitis B, were excluded.

The study protocol was explained to the patients/patients' caregivers, and written consent was sought. Demographic data and daily vital parameters (heart rate, respiratory rate, blood pressure, and oxygen saturation) were recorded. Patients were categorized into levels of function $0-6$, based on the level of ventilatory support, consciousness, and activity levels (Table 1).

Physical function was evaluated using FSS-ICU, PFIT, and FIM.

Functional Status Score in Intensive Care Unit measures five items (rolling, supine-to-sit transfer, sitting at the edge of the bed, transfer from sit to stand, and walking) of physical function based on an individual's performance, scored on an eight-point ordinal scale of $0-7 .^{9}$

Physical Function ICU Test is a test of physical function with high clinical utility and effective predictor of functional outcomes. Shoulder flexion and knee extension strength are graded from 0 to 5 (no visible or palpable contraction-full range of motion against gravity with maximal resistance). Sit-to-stand activity is scored based on the extent of assistance needed by the patient to safely transfer from sitting to standing position. Step cadence is the number of steps per minute. The patient was allowed to use assistive device if necessary. The test was concluded if the patient was unable to clear their feet from the ground for six consecutive steps or if the patient stopped marching for $>2$ seconds. The highest score of 3 was given if the patient demonstrated ability to march for $>3$ minutes. ${ }^{10}$

Functional Independence Measure is an 18-item physical function measurement tool, with 13 motor and 5 cognitive domains. Functional Independence Measure grades functional status from total independence to total assistance on the basis of level of assistance an individual requires to perform specific tasks. ${ }^{11}$

Physiotherapy treatment involving conventional therapeutic techniques based on the level of function was administered. Physiotherapist instituting care was blinded to objective scoring on functional scales. Physiotherapy care included positioning, airway clearance techniques, range-of-motion exercises, resistance exercises, breathing techniques, incentive spirometry, and early mobilization. Positioning included frequent alteration of body position, side-to-side turning in bed, upright sitting, and proning. It also included prescription of modified postural drainage positions to aid secretion removal. If needed, manual percussions and vibration techniques were applied. Airway clearance techniques were utilized along with positioning or as a sole intervention to facilitate secretion removal. Active cycle of breathing technique (ACBT), autogenic drainage (AD), huffing, and coughing techniques were appropriately selected and utilized. For intubated patients, postural drainage followed by suctioning and appropriate body positioning was done. Range-of-motion exercises for upper and lower limbs were utilized to maintain joint function. Resistance exercises targeted major muscle groups of upper and lower limbs using body weight and free weight resistance. Breathing techniques involved pursed lip breathing, diaphragmatic breathing, and thoracic expansion exercises aimed at improving the breathing pattern and relieving symptoms of dyspnea. Incentive spirometry provided a visual feedback to encourage sustained maximal inspiration to promote lung expansion. Early mobilization refers to utilizing progressive strategies to get the patient out of bed and facilitate physical activity. Intensity of exercises depended on the patient's clinical status and tolerance, starting with low intensity and gradually increasing the intensity throughout the course of rehabilitation. Each patient received two sessions per day from the day of reference until hospital discharge. Duration of each session was approximately $15-20$ minutes. ${ }^{12,13}$ Level-wise progression of treatment was achieved according to change in functional status.

\section{Statistical Methods}

Data collected at ICU admission, ICU discharge, and hospital discharge were utilized for statistical analysis Mean score was calculated for each scale component. One-way ANOVA was used for analysis of variance at the three data points. Tukey post hoc test was implemented to find out statistically significant difference or similarity between specific groups based on data points.

\section{Results}

The total number of patients included in the study was 100 (78 males and 22 females) in the age-groups of 20-29 years $(N=7)$, $30-39$ years $(N=19), 40-49$ years $(N=20)$, and 50-60 years $(N=54)$. Fifty patients were diagnosed with neurological conditions, 20 with respiratory conditions, and 30 with medical and surgical conditions. Average length of ICU stay and post-ICU hospital stay was 12.67 and 8.68 days, respectively; and average length of hospital stay from the point of ICU admission to hospital discharge was 21.33 days (Table 2).

Statistically significant between-group difference $(p<0.001)$ was demonstrated for all pre-ambulatory (rolling $f=170.21$, supineto-sit $f=145.67$, and unsupported sitting $f=113.40$ ) and ambulatory (sit-to-stand $f=172.97$ and walking $f=122.32$ ) categories of the FSS-ICU scale. All components showed statistical difference $(p<0.001)$ between ICU admission and ICU discharge, indicating that a maximum number of patients were able to achieve independent pre-ambulatory and ambulatory activities at hospital discharge.

Similarly, statistically significant improvement was observed in all categories of PFIT (shoulder strength $f=85.97$, knee strength $f=74.44$, sit-to-stand $f=122.10$, cadence $f=107.55$, cadence count

Table 1: Level 0-6 for patient categorization based on functioning

\begin{tabular}{|c|c|c|c|c|c|c|}
\hline Level 0 & Level 1 & Level 2 & Level 3 & Level 4 & Level 5 & Level 6 \\
\hline $\begin{array}{l}\text { Patient on artificial } \\
\text { ventilator on CMV } \\
\text { mode }\end{array}$ & $\begin{array}{l}\text { Change of ventilator } \\
\text { mode (CMV to SIMV/ } \\
\text { Spontaneous) }\end{array}$ & $\begin{array}{l}\text { Patient off ventilator, } \\
\text { extubated with or } \\
\text { without } \mathrm{O}_{2} \text { support }\end{array}$ & $\begin{array}{l}\text { Patient is } \\
\text { conscious and } \\
\text { oriented with } \\
\text { or without } \mathrm{O}_{2} \\
\text { support }\end{array}$ & $\begin{array}{l}\text { Patient is } \\
\text { conscious and } \\
\text { oriented without } \\
\mathrm{O}_{2} \text { support }\end{array}$ & $\begin{array}{l}\text { Patient is full } \\
\text { cooperative, } \\
\text { able to do } \\
\text { major things } \\
\text { actively }\end{array}$ & $\begin{array}{l}\text { Patient is full } \\
\text { cooperative, } \\
\text { able to do all } \\
\text { things actively }\end{array}$ \\
\hline
\end{tabular}


Physical Function in Critically Ill Patients

Table 2: Mean scores on FSS-ICU, PFIT and FIM Scales $(n=100)$

\begin{tabular}{|c|c|c|c|c|c|}
\hline \multirow[b]{2}{*}{ SI. No. } & \multirow[b]{2}{*}{ Scale scores } & \multicolumn{3}{|c|}{ Mean (SD) } & \multirow[b]{2}{*}{$p$ value } \\
\hline & & ICU admission & ICU discharge & Hospital discharge & \\
\hline \multicolumn{6}{|c|}{ Functional Status Score in Intensive Care Unit (FSS-ICU) scores } \\
\hline 1 & FSS Rolling & $1.9(1.5)$ & $4.0(1.6)$ & $6.0(1.4)$ & \\
\hline 2 & FSS Supine to sit & $1.4(1.6)$ & $3.2(1.7)$ & $5.5(1.7)$ & \\
\hline 3 & FSS Unsupported sitting & $1.2(1.7)$ & $3.2(2.2)$ & $5.5(1.9)$ & $<0.001$ \\
\hline 4 & FSS Sit to stand & $0.1(0.5)$ & $1.5(1.6)$ & $4.7(2.5)$ & \\
\hline 5 & FSS Walking & $0.0(0.5)$ & $1.0(1.5)$ & $3.9(2.6)$ & \\
\hline \multicolumn{6}{|c|}{ Physical Function ICU Test (PFIT) scores } \\
\hline 6 & PFIT Shoulder strength & $0.6(0.5)$ & $1.2(0.5)$ & $1.7(0.5)$ & \\
\hline 7 & PFIT Knee strength & $0.6(0.5)$ & $1.1(0.6)$ & $1.6(0.6)$ & \\
\hline 8 & PFIT Sit to stand & $0.0(0.3)$ & $0.6(0.8)$ & $2.1(1.4)$ & $<0.001$ \\
\hline 9 & PFIT Cadence & $0.0(0.1)$ & $0.4(0.5)$ & $1.3(0.9)$ & \\
\hline 10 & PFIT Cadence count & $0.7(4.3)$ & $8.2(12.7)$ & $39.2(30.6)$ & \\
\hline \multicolumn{6}{|c|}{ Functional Independence Measure (FIM) scores } \\
\hline 11 & FIM Eating & $1.5(1.1)$ & $2.8(1.6)$ & $5.1(1.6)$ & \multirow{18}{*}{$<0.001$} \\
\hline 12 & FIM Grooming & $1.6(1.1)$ & $2.7(1.8)$ & $4.9(1.9)$ & \\
\hline 13 & FIM Bathing & $1.1(0.8)$ & $1.4(1.1)$ & $3.8(1.9)$ & \\
\hline 14 & FIM Dressing upper body & $1.6(1.1)$ & $3.1(1.6)$ & $5.2(1.7)$ & \\
\hline 15 & FIM Dressing lower body & $1.6(1.1)$ & $2.8(1.7)$ & $4.8(1.9)$ & \\
\hline 16 & FIM Toileting & $1.1(0.7)$ & $1.6(1.3)$ & $4.4(1.9)$ & \\
\hline 17 & FIM Bladder management & $1.3(1.3)$ & $2.4(2.1)$ & $5.1(1.9)$ & \\
\hline 18 & FIM Bowel management & $1.3(1.3)$ & $2.4(2.1)$ & $5.1(1.9)$ & \\
\hline 19 & FIM Bed chair wheelchair transfer & $1.4(0.8)$ & $2.5(1.6)$ & $5.0(2.0)$ & \\
\hline 20 & FIM Toilet transfer & $1.1(0.5)$ & $1.7(1.4)$ & $4.4(2.1)$ & \\
\hline 21 & FIM Tub shower transfer & $1.0(0.5)$ & $1.7(1.4)$ & $4.4(2.1)$ & \\
\hline 22 & FIM Walk wheelchair & $1.0(0.4)$ & $1.8(1.3)$ & $4.3(2.3)$ & \\
\hline 23 & FIM Stairs & $1.0(0.0)$ & $1.3(1.0)$ & $3.1(2.0)$ & \\
\hline 24 & FIM Comprehension & $5.1(2.4)$ & $6.5(1.0)$ & $6.7(0.6)$ & \\
\hline 25 & FIM Expression & $5.0(2.6)$ & $6.2(1.4)$ & $6.5(1.1)$ & \\
\hline 26 & FIM Social interaction & $4.9(2.6)$ & $6.0(1.7)$ & $6.3(1.3)$ & \\
\hline 27 & FIM Problem-solving & $4.8(2.6)$ & $5.9(1.8)$ & $6.3(1.3)$ & \\
\hline 28 & FIM Memory & $5.0(2.6)$ & $6.2(1.4)$ & $6.4(1.1)$ & \\
\hline
\end{tabular}

$f=111.87, p<0.001)$. Shoulder and knee strength, sit-to-stand, and cadence improved from ICU admission to ICU discharge and from ICU discharge to hospital discharge. Cadence count was nonsignificant from ICU admission to ICU discharge $(p=0.016)$ and was statistically significant $(p<0.001)$ from the point of ICU discharge to hospital discharge. Thus, both FSS-ICU and PFIT scales demonstrated similar results and were capable of identifying functional limitations during hospital stay.

With respect to self-care and transfer activities, statistically significant improvement was observed in all components of the FIM tool. Post hoc analysis revealed no change in scores at the point of ICU admission to ICU discharge for self-care activities, such as bathing $(p=0.20)$ and toileting $(p=0.03)$. However, scores for eating, grooming, and dressing upper and lower body activities were statistically significant $(p<0.001)$. Bed, chair, and wheelchair transfer activity improved significantly at each data point $(p<0.001)$. Further, nonsignificant change was observed in walking $(p=0.001)$ and stair-climbing ( $p=0.13$ ) scores. Significant improvement was observed in the cognitive domains of comprehension, expression, social interaction, memory, and problem-solving $(p<0.001)$.
All motor components of FIM self-care, sphincter control, transfers, and locomotion demonstrated statistical significance from the point of ICU discharge to hospital discharge $(p<0.001)$; cognitive domains, including comprehension ( $p=0.62)$, expression $(p=0.58)$, social interaction ( $p=0.49)$, problem-solving $(p=0.26)$, and memory ( $p=0.67)$, were statistically nonsignificant from the point of ICU discharge to hospital discharge. All 18 items for motor and cognitive domains exhibited significant statistical difference $(p<0.001)$ from the point of ICU admission to hospital discharge. Self-care activities evaluated by FIM showed low scores during ICU stay, which can be attributed to the presence of disease, patients' health status, and ICU restrictions. During the post-ICU discharge phase, a faster improvement trajectory in performance of these activities was seen, indicating improved independence in performing simple motor tasks. Ability to perform transfers independently improved greatly during post-ICU hospital stay. Transfer activities remained underachieved during the period of ICU stay, possibly due to restrictions imposed by patients' health status and ICU environment. Walking function was underperformed until ICU discharge and later showed improvement until hospital discharge. Stair-climbing activity could not be scored 
in the ICU and remained less than satisfactory at hospital discharge. The post-ICU hospital phase showed improvement in ambulation and locomotory independence of patients.

All components demonstrated a gradual improvement trajectory from the point of ICU admission to hospital discharge. Although patients improved in function with more than 50th percentile at the point of hospital discharge, large deficits in function equivalent to $40-60 \%$ were observed in one domain of each outcome measure. Physical function ICU test cadence count (49.11\%), FSS-ICU walking (56.71\%), and FIM stair climbing (44.71\%) showed less-than-satisfactory improvements at the point of hospital discharge.

\section{Discussion}

This study was undertaken to evaluate functional level of patients during ICU stay and post-ICU hospital stay. A gradual improvement in physical function was observed from the point of ICU admission until hospital discharge. Physical function measures were effective in identifying functional impairments in the ICU. The chosen scales were applicable for use throughout the length of hospital stay as residual limitations continued to exist despite discharge from the ICU and even until hospital discharge.

Patients demonstrated a similar pattern of gradual rise in physical functional level in all outcome measures throughout the period of hospitalization in our study. Pre-ambulatory physical activities of rolling in bed, supine-to-sit transfer, and unsupported sitting displayed that these activities were well performed by patients during ICU stay. Similarly, weight-bearing ambulatory activities, sit-to-stand, and walking remained underachieved during ICU stay and showed gradual linear improvement during the post-ICU hospital phase. It can thus be said that applicability of FSS-ICU is a sensitive measure that can be used to objectively record improvement in preambulatory and ambulatory activities.

Loss of muscle strength is known to be acquired in critically ill patients who are mechanically ventilated for prolonged time periods. Studies show that ICU-acquired weakness (ICU-AW) is present in more than $50 \%$ of patients at ICU discharge who were mechanically ventilated for more than 48 hours. Significant muscle loss due to both bed rest and the presence of critical illness results in decreased ability to perform self-care and transfer activities and worsening of disability in ICU survivors. ICU-acquired weakness manifests even after discharge from acute healthcare centers as post-intensive care syndrome (PICS), resulting in impairment in physical functioning, cognitive dysfunction, and poor mental health status. Symptoms include generalized weakness, fatigue, decreased mobility, anxious or depressed mood, sexual dysfunction, sleep disturbances, and cognitive issues (memory disturbance/loss, slow mental processing, poor concentration, and so on) and can last for a few months to many years post-recovery. Physiotherapy interventions focused on exercise-based interventions in the ICU, including both bed exercises and mobilization out of bed, can help reduce incidence of ICU-AW and PICS. ${ }^{14}$ Therefore, identification of these functional disabilities gains utmost importance prior to hospital discharge.

The physical function ICU test (PFIT) tool provides an objective measure of improvement in muscle strength of shoulder flexors and knee extensors; both these muscles groups showed gradual improvement from the point of ICU admission to ICU discharge and further up to hospital discharge. This supports effectiveness of adding resistive exercises in the treatment protocol of ICU patients. By identification of muscle weakness, physiotherapy interventions focusing on the management of the same can be utilized early in rehabilitation of ICU patients.

Results of our study supported the effectiveness of physiotherapy interventions and their effects on physical function post-ICU discharge. After ICU discharge, considerable improvement in physical activity and functional level status of patients was demonstrated on FIM. Self-care activities, such as eating, grooming, dressing, and toileting, showed greatest improvement in independence during and after ICU discharge. Level of physical activity displayed a steady increase during and after ICU discharge, with patients performing tasks of self-care and bed transfers much more independently than during ICU stay. Walking and stairclimbing activities remained underachieved at hospital discharge, achieving less than $60 \%$ improvement. FSS-ICU, PFIT, and FIM were useful for identifying limitations in walking and stair-climbing functions at the point of hospital discharge. The results from this study emphasize the need to monitor impaired cardiorespiratory function, muscle weakness, and malnutrition on a long-term basis as these are important factors for poor ambulatory performance after ICU discharge. ${ }^{15}$ Similar observations are reported in previous studies conducted on individuals admitted to ICU for $\geq 72$ hours. ${ }^{16,17}$

Difficulty in performing ADLs, transfers, and ambulation is commonly encountered by ICU survivors. ICU survivors with a low burden of preexisting comorbidities have little to no disability at hospital discharge and generally show good outcomes. ${ }^{18}$ Higher test scores of FSS-ICU, PFIT, and FIM at ICU discharge can be used as significant factors in determining discharge to home. ${ }^{19,20}$

Out of all the outcome measures, only FIM scale evaluated cognitive aspects, including comprehension, expression, social interaction, problem-solving, and memory. All patients showed improvement in cognitive function from the point of ICU admission up to hospital discharge. Higher physical activity level aided achievement and maintenance of good cognitive functions during hospital stay. These findings support previous studies that conclude early rehabilitation of patients in the form of physical activity in the ICU improves cognitive function and helps to reduce symptoms of anxiety and depression. Early rehabilitation thus decreases risk of neuropsychiatric morbidities in critically ill patients.

Through this study, we were able to recognize barriers to early mobilization of ICU patients that prevented achieving higher levels of mobility during ICU stay. Dafoe et al. categorized these barriers as patient-related and institution-related barriers. Patient-related barriers include medical instability, sedation, level of consciousness, and the presence of vascular lines/monitor attachments. Institutionrelated barriers include poor staff awareness of the benefits of early progressive mobilization, resulting in a conservative culture where mobilization is undervalued, uncertainty regarding staff responsibility for mobilization (e.g., physiotherapists or nurses), and insufficient staff/equipment. ${ }^{21}$ Patient barriers play a major role in lack of achieving ambulatory activities in the ICU. After ICU discharge, patients are exposed to greater functional freedom to perform physical activities with reduced or no barriers.

Our study demonstrated an upward trend in the physical functional trajectory of patients during ICU stay and post-ICU hospital stay. However, residual physical function limitations continue to persist at the point of hospital discharge, necessitating continuum of care. Thus, by informing caregivers regarding these 
limitations, strategies can be adopted to combat and help overcome long-term morbidity in these patients.

\section{Conclusion}

Objective assessment of the physical function trajectory helps to monitor and understand patients' level of functional status throughout their hospital stay. It helps in gaining better insight of patients' limitations to functional activities and can help in goalsetting and planning of physiotherapy care.

Early and progressive rehabilitation intervention programs are feasible for comprehensive management of ICU patients; this reduces physical and mental health complications that frequently occur in survivors of critical illnesses. Early physical rehabilitation benefits patients in domains of muscle strength, physical function, and quality of life, which helps in recovering optimum physical function and independence in daily life. Greater standardization of physiotherapy management of patients in the ICU can be achieved by continuing rehabilitation until hospital discharge and follow-up rehabilitation after hospital discharge.

Implementing and continuing early rehabilitation physiotherapy until hospital discharge and later implementing home rehabilitation after hospital discharge will help patients to recover optimum physical function and independence in activities of daily living.

\section{Clinical Implication}

Identification of functional limitations aids in prescribing a targeted treatment approach for patients starting early in the ICU, continuing throughout hospital stay and beyond hospital discharge.

\section{Limitations}

Our study included a wide variety of patients with neurological, respiratory, medical, and surgical conditions. Further research on patients admitted to various other ICUs with specific conditions needs to be undertaken for wider applicability of observations from this study.

\section{ORCID}

Damini R Aglawe 으 https://orcid.org/0000-0003-4986-8903

Bela M Agarwal @ https://orcid.org/0000-0001-7573-9844

Bhoomika D Sawant ๑ https://orcid.org/0000-0001-6856-8880

\section{References}

1. Topp R, Ditmyer M, King K, Doherty K, Hornyak J III. The effect of bed rest and potential of prehabilitation on patients in the intensive care unit. AACN Clin Issues 2002;13(2):263-276. DOI: 10.1097/00044067200205000-00011.

2. Perme C, Chandrashekar R. Early mobility and walking program for patients in intensive care units: creating a standard of care. Am J Crit Care 2009;18(3):212-221. DOI: 10.4037/ajcc2009598.

3. Akima H, Kubo K, Kanehisa H, Suzuki Y, Gunji A, Fukunaga T. Legpress resistance training during 20 days of 6 head-down-tilt bed rest prevents muscle deconditioning. Eur J Appl Physiol 2000;82(1-2): 30-38. DOI: 10.1007/s004210050648.

4. Convertino VA. Cardiovascular consequences of bed rest: effect on maximal oxygen uptake. Med Sci Sports Exerc 1997;29(2):191-196. DOI: 10.1097/00005768-199702000-00005.
5. Shoemaker JK, Hogeman CS, Sinoway LI. Contributions of MSNA and stroke volume to orthostatic intolerance following bed rest. Am J Physiol Regul Integr Compar Physiol 1999;277(4):R1084-R1090. DOI: 10.1152/ajpregu.1999.277.4.r1084.

6. Parry SM, Huang M, Needham DM. Evaluating physical functioning in critical care: considerations for clinical practice and research. Crit Care 2017;21(1):249. DOI: 10.1186/s13054-017-1827-6.

7. Needham DM. Mobilizing patients in the intensive care unit: improving neuromuscular weakness and physical function. Journal of the American Medical Association 2008;300(14):1685-1690. DOI: 10.1001/jama.300.14.1685.

8. Parry SM, Knight LD, Connolly B, Baldwin C, Puthucheary Z, Morris P, et al. Factors influencing physical activity and rehabilitation in survivors of critical illness: a systematic review of quantitative and qualitative studies. Intensive Care Med 2017;43(4):531-542. DOI: 10.1007/s00134-017-4685-4.

9. Hiser S, Toonstra A, Friedman LA, Colantuoni E, Connolly B, Needham DM. Interrater reliability of the functional status score for the intensive care unit. J Acute Care Phys Ther 2018;9(4):186-192. DOI: 10.1097/JAT.0000000000000086.

10. Denehy L, de Morton NA, Skinner EH, Edbrooke L, Haines K, Warrillow $\mathrm{S}$, et al. A physical function test for use in the intensive care unit: validity, responsiveness, and predictive utility of the physical function ICU test (scored). Phys Ther 2013;93(12):1636-1645. DOI: 10.2522/ptj.20120310.

11. Gosman-Hedström G, Svensson E. Parallel reliability of the functional independence measure and the Barthel ADL index. Disabil Rehabil 2000;22(16):702-715. DOI: 10.1080/09638280050191972.

12. Frownfelter D, Dean E. Cardiovascular and pulmonary physical therapy-E-Book: evidence to practice. Elsevier Health Sci; 2014.

13. Thomson AM, Skinner AT, Piercy J. Tidy's physiotherapy. ButterworthHeinemann; 1991.

14. Hodgson $\mathrm{CL}$, Tipping CJ. Physiotherapy management of intensive care unit-acquired weakness. J Physiother 2017;63(1):4-10. DOI: 10.1016/j.jphys.2016.10.011.

15. Da Silva PB, Dos Santos LJ. Patient functionality and walking speed after discharge from the intensive care unit. Rev Bras Ter Intensiva 2019;31(4):529. DOI: 10.5935/0103-507X.20190066. ISBN: 9780323291170.

16. Vesz PS, Costanzi M, Stolnik D, Dietrich C, Freitas KL, Silva LA, et al. Functional and psychological features immediately after discharge from an intensive care unit: prospective cohort study. Rev Bras Ter Intensiva 2013;25(3):218-224. DOI: 10.5935/0103507X.20130038.

17. Van der Schaaf M, Dettling DS, Beelen A, Lucas C, Dongelmans DA, Nollet F. Poor functional status immediately after discharge from an intensive care unit. Disabil Rehabil 2008;30(23):1812-1818. DOI: 10.1080/09638280701673559.

18. Shimogai T, Izawa KP, Kawada M, Kuriyama A. Factors affecting discharge to home of medical patients treated in an intensive care unit. Int J Environ Res Public Health 2019;16(22):4324. DOI: 10.3390/ ijerph16224324.

19. Tymkew H, Norris T, Arroyo C, Schallom M. The use of physical therapy ICU assessments to predict discharge home. Crit Care Med 2020;48(9):1312-1318. DOI: 10.1097/CCM.0000000000004467.

20. Parry SM, Denehy L, Beach LJ, Berney S, Williamson HC, Granger CL. Functional outcomes in ICU-what should we be using? An observational study. Crit Care 2015;19(1):127. DOI: 10.1186/s13054015-0829-5.

21. Dafoe S, Chapman MJ, Edwards S, Stiller K. Overcoming barriers to the mobilisation of patients in an intensive care unit. Anaesth Intens Care 2015;43(6):719-727. DOI: 10.1177/0310057X1504300609. 